\title{
Отрицательная дифференциальная проводимость структур на основе оксида лантана
}

\author{
(C) А. Игитян ${ }^{1,2}$, Н. Агамалян ${ }^{1,2}$, Р. Овсепян ${ }^{1,2}$, С. Петросян ${ }^{1,2}$, Г. Бадалян ${ }^{1}$, \\ И. Гамбарян ${ }^{1}$, А. Папикян ${ }^{2}$, Е. Кафрадарян ${ }^{1,2, \text { Ф }}$ \\ ${ }^{1}$ Институт фризических исследований Национальной академии наук Армении, \\ 0203 Аштарак, Армения \\ 2 Российско-Армянский университет, \\ 0051 Ереван, Армения \\ "E-mail: ekafadaryan@gmail.com \\ Поступила в Редакцию 7 октября 2019 г. \\ В окончательной редакции 15 октября 2019 г. \\ Принята к публикации 15 октября 2019 г.
}

\begin{abstract}
С помощью метода электронно-лучевого напыления получены прозрачные поверхностно-гидрированные пленки оксида лантана $\left(\mathrm{OH}-\mathrm{La}_{2} \mathrm{O}_{3}\right)$ толщиной 40,140 и 545 нм. Исследованы электрические и оптические характеристики структур $\mathrm{Al} / \mathrm{OH}-\mathrm{La}_{2} \mathrm{O}_{3} / p-\mathrm{Si}$, где в качестве верхнего и нижнего электродов использовали соответственно алюминий и кремниевую подложку с $p$-типом проводимости. Обнаружена область отрицательной дифференциальной проводимости на зависимостях проводимости от напряжения при прямом смещении; возможный механизм отрицательной дифференциальной проводимости объясняется переносом протонов по цепочкам молекул воды, связанных водородными связями на поверхности пленки $\mathrm{OH}-\mathrm{La}_{2} \mathrm{O}_{3}$.
\end{abstract}

Ключевые слова: отрицательная дифференциальная проводимость, $\mathrm{OH}-\mathrm{La}_{2} \mathrm{O}_{3}$, протонная проводимость.

DOI: $10.21883 / F T P .2020 .02 .48915 .9280$

\section{1. Введение}

Среди материалов с высокой диэлектрической проницаемостью, рассматриваемых в качестве подзатворного слоя толщиной $\sim 1$ нм в тонкопленочных нанотранзисторах, для замены диоксида кремния $(\varepsilon=4.5)$ наиболее подходящим является гексагональный оксид лантана $\left(h-\mathrm{La}_{2} \mathrm{O}_{3}\right)$ из-за высокой диэлектрической проницаемости $(\varepsilon=27)[1,2]$, высокой напряженности электрического пробоя [3], термостойкости на кремниевой подложке [4], относительно большого смещения зон по отношению к зонам кремния [2,5] и прозрачности в диапазоне длин волн от ультрафиолета (УФ) до инфракрасного (ИК) [6]. В этой связи подробно исследованы структурные, оптические и электрические характеристики $h$ - $\mathrm{La}_{2} \mathrm{O}_{3}$ [1-6]. Кроме того, в структурах на основе $\mathrm{La}_{2} \mathrm{O}_{3}\left(\mathrm{TiN} / \mathrm{La}_{2} \mathrm{O}_{3} / \mathrm{PtTi} / \mathrm{SiO}_{2} / \mathrm{Si}, \mathrm{Pt} / \mathrm{La}_{2} \mathrm{O}_{3} / \mathrm{Pt}\right)$ выявлена резистивная память с биполярным переключением, разрабатываемая для создания энергонезависимой резистивной памяти (RRAM) [7,8].

Хорошо известно, что пленки $\mathrm{La}_{2} \mathrm{O}_{3}$ гигроскопичны, притягивают и удерживают молекулы воды из окружающей среды на поверхности, что влияет на транспортные свойства оксида лантана [9]. Как было показано нами в работе [10], структуры на основе поверхностно-гидрированных пленок оксида лантана $\left(\mathrm{OH}-\mathrm{La}_{2} \mathrm{O}_{3}\right)$, напыленных на кремниевые подложки с $n$-типом проводимости $\left(\mathrm{Al} / \mathrm{OH}-\mathrm{La}_{2} \mathrm{O}_{3} / n-\mathrm{Si}\right)$, имеют отрицательную дифференциальную проводимость (ОДП), когда увеличение напряжения приводит к уменьшению тока. В данной работе этот эффект становится более заметным в пленках $\mathrm{OH}-\mathrm{La}_{2} \mathrm{O}_{3}$ на кремниевых под- ложках с дырочной проводимостью (p-Si). Исследованы зависимости проводимости и емкости от напряжения структур $\mathrm{Al} / \mathrm{OH}-\mathrm{La}_{2} \mathrm{O}_{3} / p$ - $\mathrm{Si}$, где наличие участка с отрицательной дифференциальной проводимостью объясняется переносом протона по цепочке связанных водородными связями молекул воды. ОДП, обусловленная переносом протонов, представляет интерес, поскольку положительно заряженные ионы $\left(\mathrm{H}^{+}\right)$имеют более высокую подвижность, чем подвижность ионов кислорода $\left(\mathrm{O}^{2-}\right)$. В этой связи использование протонной памяти может улучшить скорость резистивного переключения оперативной памяти RRAM по сравнению с типичными RRAM (мемристорами), основанными на миграции ионов кислорода. ОДП является активным компонентом для построения логических схем, диодов, памяти RRAM, переключателей и датчиков, и представленные здесь данные могут потенциально использоваться для разработки RRAM, используемых в цифровых и аналоговых интегральных микросхемах.

\section{2. Экспериментальная часть}

Пленки $\mathrm{OH}-\mathrm{La}_{2} \mathrm{O}_{3}$ толщиной 40, 140 и 545 нм получены на кварцевой $\left(\mathrm{SiO}_{2}\right)$ и кремниевой подложке с $p$-типом проводимости $(p-\mathrm{Si})$ методом электроннолучевого напыления. Мишени для напыления изготавливались методом твердофазного синтеза. Порошок окисида лантана 99\% чистоты прессовался при давлении

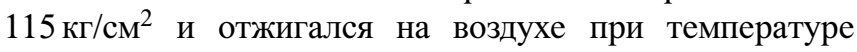
$1430^{\circ} \mathrm{C}$ в течение 120 мин. Пленки $\mathrm{OH}-\mathrm{La}_{2} \mathrm{O}_{3}$ напылялись при температуре $250^{\circ} \mathrm{C}$. Вакуумная система 


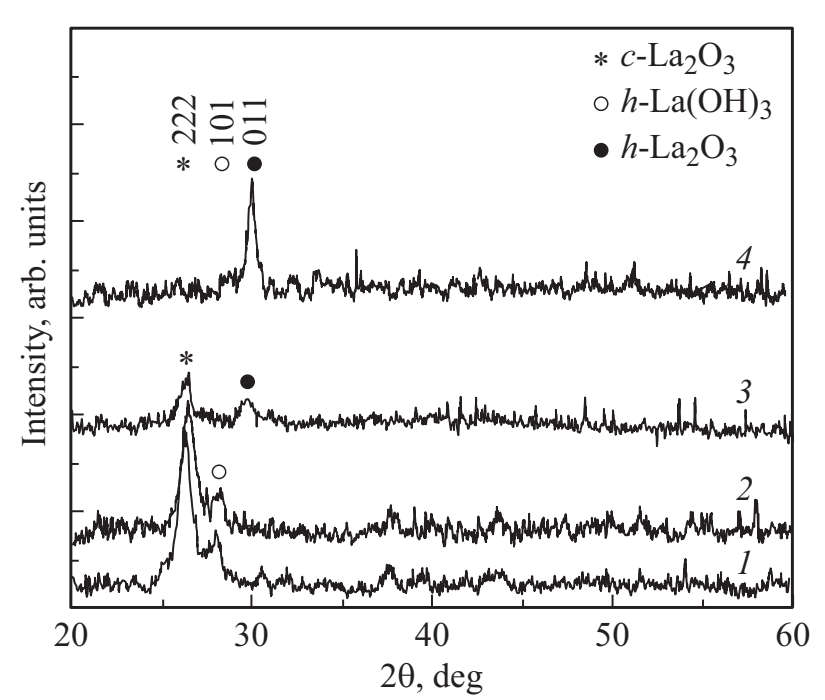

Рис. 1. Рентгенограммы пленки $\mathrm{OH}-\mathrm{La}_{2} \mathrm{O}_{3}$ толщиной 545 нм на кварцевой подложке $\left(\mathrm{SiO}_{2}\right)$ после напыления $(1)$, хранения в вакууме (2) и отжига (3); рентгенограмма пленки $\mathrm{OH}-\mathrm{La}_{2} \mathrm{O}_{3}$ толщиной 140 нм после напыления (4).

откачки обеспечивала вакуум $\sim 10^{-5}$ Торр. Время напыления составляло 5-20 мин в зависимости от желаемой толщины. Скорость нанесения пленки составляла $60 \AA / \mathrm{c}$.

Структурные, оптические и электрические измерения пленок проводились сразу после изьятия из напылительной установки. Далее пленки хранились в низковакуумной камере с давлением $5 \cdot 10^{-2}$ Торр. После хранения в вакууме пленки отжигались при $650^{\circ} \mathrm{C}$ в течение 60 мин в вакууме ( $\left.10^{-2} \mathrm{Topp}\right)$. Пленку $\mathrm{OH}-\mathrm{La}_{2} \mathrm{O}_{3}$ толщиной 40 нм подвергали вакуумному отжигу при $500^{\circ} \mathrm{C}$ в течение 15 мин без предварительного выдерживания в вакуумной камере. Фазовый состав пленок изучали с помощью рентгеновской дифракции (XRD) в геометрии Брэгга-Брентано с $\mathrm{Cu} K_{\alpha}(\lambda=1.5418 \AA)$ излучением (Дрон-4). Морфологию поверхности и элементный состав образцов исследовали на сканирующем электронном микроскопе (SEM VEGA TS-5130MM) с системой рентгеновского энергодисперсионного микроанализа (EDS) INCA Energy 300 с энергией электронного зонда 14, 18 и 20 кэВ. Инфракрасный спектрофотометр Specord M-80 (Carl Ziess Jena) использовался для регистрации гидроксильных групп. Ширину запрещенной зоны пленок определяли с помощью UV-VIS-IR спектрофотометров Specord M-40 и СФ-8 (ЛОМО). Толщину пленки определяли методом оптической интерференции, SEM и профилометром Ambios XP-1. Измерения проводимость-напряжение $(\sigma-V)$ и емкость-напряжение $(C-V)$ образцов проводились на частоте 1 кГц при напряжениях смещения от -10.0 до +10.0 В с помощью цифрового RLC-метра E7-8 (Россия). Верхние алюминиевые (Al) электроды площадью $5 \cdot 10^{-3} \mathrm{~cm}^{2}$ напылялись методом термического испарения.

\section{3. Результаты и обсуждение}

Рентгенограммы пленок $\mathrm{OH}-\mathrm{La}_{2} \mathrm{O}_{3}$ демонстрируют кристаллическую структуру. Как видно из рис. 1, пленка толщиной 545 нм (спектр 1) содержит кубический $\left(c-\mathrm{La}_{2} \mathrm{O}_{3}\right)$ (JCPDS № 04-0856) и гексагональный гидроксид лантана $h-\mathrm{La}(\mathrm{OH})_{3}$ (JCPDS № 83-2034). Хранение пленки в низковакуумной камере в течение 10 и 120 дней не влияет на рентгеновские дифракционные спектры (спектр 2). После вакуумного отжига при $650^{\circ} \mathrm{C}$ в течение 1 ч пик (101), соответствующий $\mathrm{La}(\mathrm{OH})_{3}$, исчезает (спектр 3), кубический (222) пик уменьшается по интенсивности и появляется низкоинтенсивный (011) рефлекс, который соответствует гексагональной фазе $h-\mathrm{La}_{2} \mathrm{O}_{3}$ (JCPDS № 83-1355). Согласно нашим результатам, пленка толщиной 545 нм состоит из смеси кубической и гексагональной фаз, тогда как пленка толщиной 140 нм демонстрирует гексагональную симметрию (рис. 1, спектр 4).

Отношение сигнал/шум на рентгенограмме пленки толщиной 40 нм низкое, поэтому состав пленки опреде-

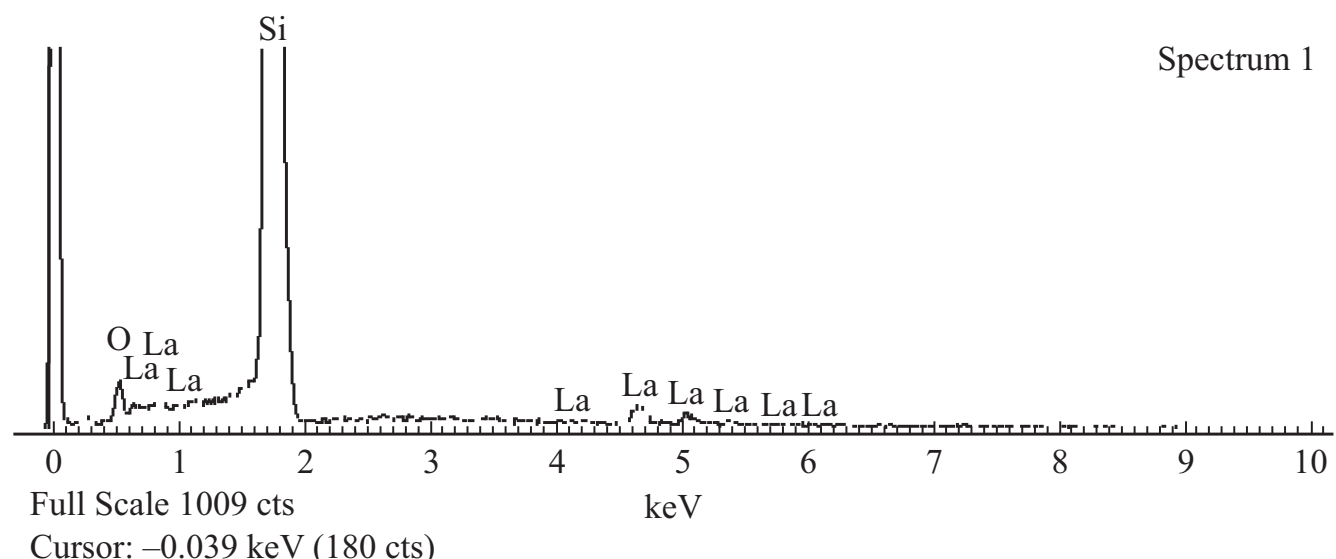

Pис. 2. EDS пленки $\mathrm{OH}-\mathrm{La}_{2} \mathrm{O}_{3}$ толщиной 40 нм на кремниевой подложке. 


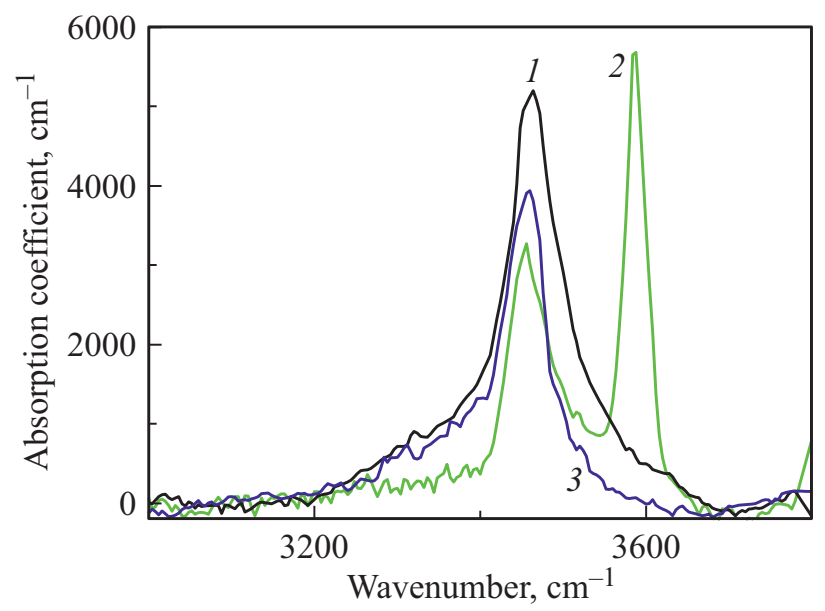

Рис. 3. ИК-спектры пленки $\mathrm{OH}-\mathrm{La}_{2} \mathrm{O}_{3}$ толщиной 140 нм на кварцевой подложке после напыления (1), хранения в вакууме (2) и отжига (3).

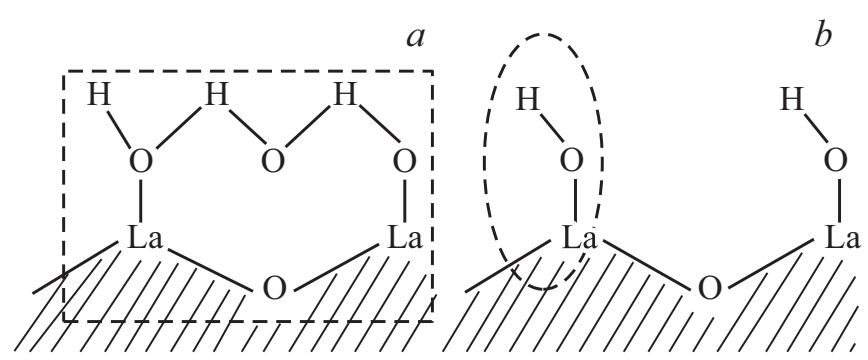

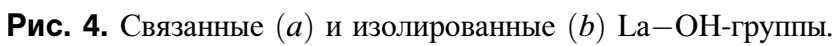

ляли с помощью EDS с энергией зонда 14 кэВ. На рис. 2 спектр EDS демонстрирует пики O и La с отношением атомных концентраций $\mathrm{O} / \mathrm{La}=2$. Отношение $\mathrm{O} / \mathrm{La}$ пле- нок толщиной 140 и 545 нм находится в области $1.7-2.2$. Легкие элементы, такие как водород, не могут быть зарегистрированы с помощью EDS, поэтому наличие гидроксильных групп в пленках $\mathrm{OH}-\mathrm{La}_{2} \mathrm{O}_{3}$ определяли методом ИК-спектроскопии. На рис. 3 представлены ИК-спектры поглощения пленки $\mathrm{OH}-\mathrm{La}_{2} \mathrm{O}_{3}$ толщиной 140 нм на кварцевой подложке после напыления (спектр 1), хранения в вакууме (спектр 2) и отжига (спектр 3).

В результате поглощения воды из окружающей среды наблюдается полоса $3458 \mathrm{~cm}^{-1}$, соответствующая

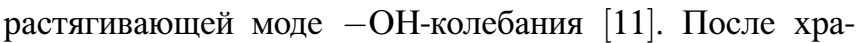
нения пленок в низковакуумной среде при комнатной температуре возникает новая полоса при $3586 \mathrm{~cm}^{-1}$ (спектр 2), которая исчезает при отжиге (спектр 3). Эти полосы интерпретируются как свободные $\left(3586 \mathrm{~cm}^{-1}\right)$ и связанные $\left(3458 \mathrm{~cm}^{-1}\right)$ колебания -OН-группы, атом кислорода которых координирован с поверхностными ненасыщенными ионами лантана (рис. 4). Аналогичные результаты получены для пленок толщиной 40 и 545 нм. Спектры оптического диапазона пленок $\mathrm{OH}-\mathrm{La}_{2} \mathrm{O}_{3}$ на подложках $\mathrm{SiO}_{2}$ имеют высокий коэффициент пропускания $(90-80 \%)$ с оптическим краем, чувствительным к толщине пленки и условиям обработки [10]. Результаты показали, что ширина запрещенной зоны $E_{g}=6.12$ эВ уменьшается до 6.06 эВ с увеличением толщины пленки от 140 до 545 нм, тогда как толщина пленок после хранения в вакууме увеличивается по сравнению с толщиной только напыленной пленки, и уменьшается после термического отжига (см. таблицу).

Зависимости проводимости от напряжения $(\sigma-V)$ структур $\mathrm{Al} / \mathrm{OH}-\mathrm{La}_{2} \mathrm{O}_{3} / p$-Si измерены в диапазоне напряжений $0 \rightarrow 10 \mathrm{~B} \rightarrow 0 \mathrm{~B} \rightarrow-10 \mathrm{~B} \rightarrow 0 \mathrm{~B}$ на частоте 1 кГц. $\sigma-V$-характеристики трех пленок $\mathrm{OH}-\mathrm{La}_{2} \mathrm{O}_{3}$, измеренные после напыления, имеют гистерезис и участок
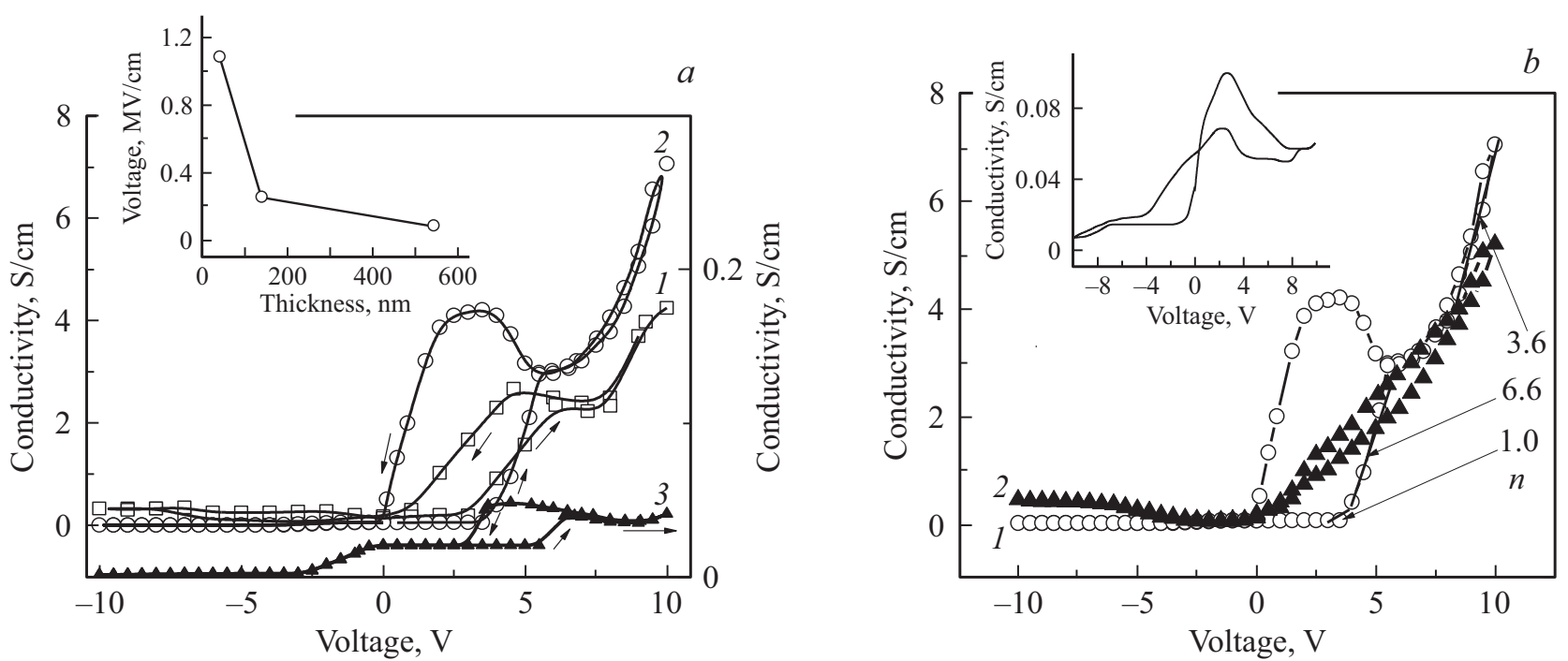

Рис. 5. $a$ - зависимости проводимости от напряжения $(\sigma-V)$-структур $\mathrm{Al} / \mathrm{OH}-\mathrm{La}_{2} \mathrm{O}_{3} / p-\mathrm{Si}$ с толщиной пленок $\mathrm{OH}-\mathrm{La}_{2} \mathrm{O}_{3}$ $40(1), 140$ (2) и 545 (3) нм после напыления (на вставке представлена зависимость $V_{\operatorname{Pr}}$ от толщины), $b-\sigma-V$-зависимость $\mathrm{Al} / \mathrm{OH}-\mathrm{La}_{2} \mathrm{O}_{3} / \mathrm{Si}-p$ после напыления, хранения в вакууме и отжига (вставка) пленки толщиной 140 нм. 
Ширина запрещенной зоны $\left(E_{g}\right)$, толщина $(d)$ и частота колебаний $-\mathrm{OH}-г$ упп пленок $\mathrm{OH}-\mathrm{La}_{2} \mathrm{O}_{3}$ толщиной 140 и 545 нм после напыления, хранения в вакууме и отжига [10]

\begin{tabular}{l|c|c|c|c|c}
\hline & $d$, нм & $E_{g}$, эВ & $d$, нм & $E_{g}$, эВ & $f_{\text {он, } \mathrm{cm}^{-1}}$ \\
\hline После напыления & 140 & 6.12 & 545 & 6.06 & 3450 \\
После хранения & 160 & 5.92 & 635 & 5.903 & 3450, \\
в вакууме & & & & & 3586 \\
После отжига & 150 & 6.04 & 560 & 5.958 & 3450
\end{tabular}

ОДП при изменении положительной ветви напряжения от 0 до 10 В и выпрямлямление тока при сканировании от 0 до $-10 \mathrm{~B}$ (рис. 5, $a$ ). Отношение максимального значения проводимости к минимальному $\left(\sigma_{\max } / \sigma_{\min }\right)$ петли гистерезиса равно 14,80 и 2.6 соответственно для пленок толщиной 40, 140 и 545 нм. После хранения пленок в низковакуумной камере $\sigma-V$-характеристики представляют собой обычную экспоненциальную зависимость без ОДП (рис. 5, b). ОДП вновь появляется после отжига при $650^{\circ} \mathrm{C}$ в вакууме (см. вставку на рис. $\left.5, b\right)$. Можно заметить корреляцию между электрическими и оптическими характеристиками $\mathrm{Al} / \mathrm{OH}-\mathrm{La}_{2} \mathrm{O}_{3} / p-\mathrm{Si}$ структур. Появление ОДП на кривых $\sigma-V$ ассоциируется с пиком поглощения при $3458 \mathrm{~cm}^{-1}$, тогда как исчезновение ОДП сопровождается появлением дополнительного пика при $3586 \mathrm{~cm}^{-1}$. Для количественной дифференциации изолированных и связанных -ОН-групп вычислены площади под пиками, используя форму спектральной линии Лоренца. Площадь пика при $3458 \mathrm{~cm}^{-1}$ между 3000-3800 $\mathrm{cm}^{-1}$ составляет $6.38 \cdot 10^{5} \mathrm{~cm}^{-2}$ после напыления (спектр 1). Появление пика при $3586 \mathrm{~cm}^{-1}$ площадью $2.0 \cdot 10^{5} \mathrm{~cm}^{-2}$ сопровождается уменьшением пика $3458 \mathrm{~cm}^{-1}$ до площади $2.6 \cdot 10^{5} \mathrm{~cm}^{-2}$ и исчезновением ОДП, что можно объяснить разрывом водородных связей из-за тепловых флуктуаций. После отжига при $650^{\circ} \mathrm{C}$ появляется участок ОДП, и площадь пика при $3458 \mathrm{~cm}^{-1}$ увеличивается до $4.03 \cdot 10^{5} \mathrm{~cm}^{-2}$, т. е. низковауумный отжиг увеличивает количество связанных -ОН-групп. Согласно литературным данным [12], ОДП обычно основана на квантовом туннелировании и междолинном переносе носителей заряда. В данном случае туннелирование не является причиной возникновения ОДП, поскольку ОДП в $\mathrm{Al} / \mathrm{OH}-\mathrm{La}_{2} \mathrm{O}_{3} / p$-Si сильно зависит от направления развертки напряжения. Согласно литературным данным [13-17], ОДП может возникнуть в результате бистабильного межфазного переключения, окислительно-восстановительной реакции и протонопосредованных механизмов. Во всех этих механизмах бистабильные состояния обусловлены химическими реакциями. Например, при бистабильном межфазном переключении ОДП происходит в результате перехода от сильного к слабому химическому связыванию [13-16].
В случае окислительно-восстановительного механизма ОДП происходит за счет окисления и восстановления активных молекул при определенном напряжении [17]. Происхождение ОДП в структурах $\mathrm{Al} / \mathrm{OH}-\mathrm{La}_{2} \mathrm{O}_{3} / p-\mathrm{Si}$ можно объяснить протонной проводимостью, возникающей в результате диссоциации воды $\left(\mathrm{H}_{2} \mathrm{O} \leftrightarrow \mathrm{H}^{+}+\mathrm{OH}^{-}\right)$ на поверхности пленки. Молекулы воды вблизи анода в результате электролиза диссоциируют на протон $\mathrm{H}^{+}$и гидроксильную группу $\mathrm{OH}^{-}$, а быстрый дрейф протонов [18] к катоду и медленное движение $\mathrm{OH}^{-}$к месту диссоциации, где число протонов убывает, инициирует ОДП. Кроме того, согласно прыжковому механизму Гротта [19], протон в форме иона гидрония $\left(\mathrm{H}_{3} \mathrm{O}^{+}\right)$ может мигрировать вдоль границ зерен, двигаясь к катоду по цепочкам молекул воды, связанных водородными связями. Этот механизм включает также транспорт $\mathrm{OH}^{-}$, рассматриваемый в качестве ,протонной дыры“ и имеет важное значение для объяснения протонного переноса как на поверхности, так и на границах зерен пленки $\mathrm{OH}-\mathrm{La}_{2} \mathrm{O}_{3}$.

Для лучшего понимания механизма проводимости проведено фитирование кривых $\sigma-V$ в соответствии с ТОПЗ (ток, ограниченный пространственным зарядом), эмиссией Шоттки $\ln I \propto V^{1 / 2}$, эмиссией Пула-Френкеля $\ln (I / V) \propto V^{1 / 2}$ и туннелированием Фаулера-Нордгейма $\ln \left(I / V^{2}\right) \propto(1 / V)[1-5,20,21]$. Фитирование вольт-амперных кривых показало, что доминирующим механизмом проводимости $\mathrm{Al} / \mathrm{OH}-\mathrm{La}_{2} \mathrm{O}_{3} / p-\mathrm{Si}$ является ТОПЗ ( $I \sim V^{n}, n-$ наклон кривой). На рис. 5, $b$ показаны области, где значение наклона $n=1$ (закон Ома) в области $V<3.6 \mathrm{~B}$ увеличивается до 6.6 в области $3.6<V<5.6 \mathrm{~B}$ и 3.6 при $V>7 \mathrm{~B}$, что соответствует ТОПЗ, когда все ловушки оказываются заполненными [22]. Вольт-амперные характеристики образцов, хранящихся в вакууме, следовали закону Чайлда $(n=2)$. Протонный вклад в общую проводимость устройств обнаруживается при определенном напряжении $\left(V_{\mathrm{Pr}}\right)$, соответствующем максимуму тока, после которого ток уменьшается при увеличении напряжения. Как видно на вставке рис. 5,a, напряженность $V_{\mathrm{Pr}}^{\prime}$ уменьшается с увеличением толщины пленки и составляет $1.2 \cdot 10^{6}, 0.23 \cdot 10^{6}$ и $0.08 \cdot 10^{6} \mathrm{~B} / \mathrm{cm}$ для пленок соответственно толщиной 40, 140 и 545 нм. Таким образом, для разрыва водородных связей в толстых пленках требуются меньшие напряжения, чем в тонких пленках.

На рис. 6 приведены вольт-фарадные (ВФХ) характеристики $(C-V)$ структур $\mathrm{Al} / \mathrm{OH}-\mathrm{La}_{2} \mathrm{O}_{3} / p-\mathrm{Si}$ с разными толщинами пленок $\mathrm{OH}-\mathrm{La}_{2} \mathrm{O}_{3}$. Зависимости $C-V$ характерны для устройств с нелинейной емкостью и имеют гистерезис, который указывает на наличие подвижных ионов. $C-V$-характеристики структур $\mathrm{Al} / \mathrm{OH}-\mathrm{La}_{2} \mathrm{O}_{3} / p-\mathrm{Si}$ показывают положительный сдвиг напряжения плоских зон, свидетельствующий о наличии отрицательного встроенного заряда. Для оценки количества подвижных ионов $N_{S S}$ (поверхностная плотность) используется сле- 


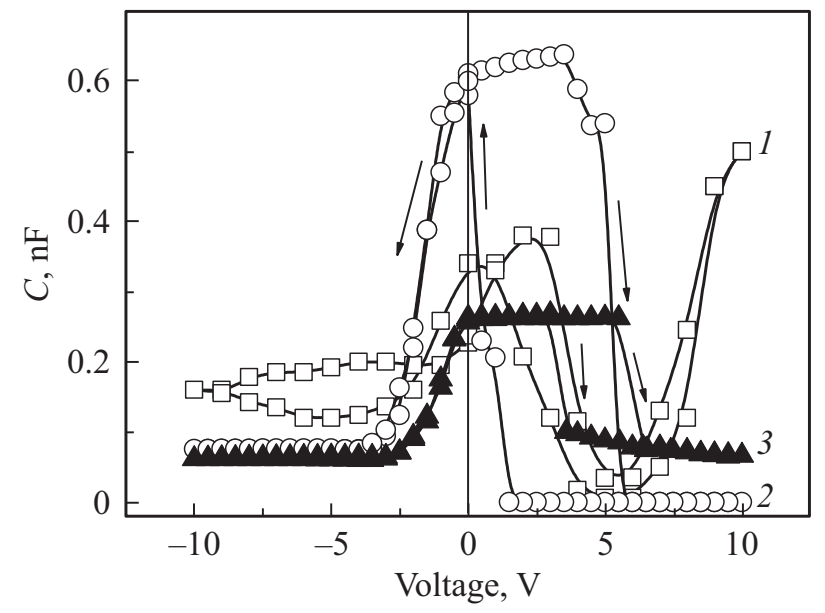

Рис. 6. $\mathrm{C}-\mathrm{V}$-характеристики $\mathrm{Al} / \mathrm{OH}-\mathrm{La}_{2} \mathrm{O}_{3} / p-\mathrm{Si}$ структур с толщинами пленок $\mathrm{OH}-\mathrm{La}_{2} \mathrm{O}_{3} 40$ (1), 140 (2) и 545 (3) нм.

дующее выражение [23]:

$$
N_{S S}=\frac{\delta V C_{\max }}{e S}
$$

где $S$ - площадь верхнего электрода, $\delta V-$ величина гистерезиса, $C_{\max }-$ максимальная емкость, $e-$ заряд электрона. $N_{s s}$ пленок толщиной 40,140 и 540 нм составляет соответственно $1.2 \cdot 10^{12}, 5.5 \cdot 10^{12}$ и $0.9 \cdot 10^{12} \mathrm{~cm}^{-2}$.

Диэлектрическая проницаемость рассчитывается на основе формулы емкости конденсатора с параллельными пластинами как

$$
C_{o x}=\frac{\varepsilon \varepsilon_{0} A}{d_{o x}},
$$

где $\varepsilon-$ диэлектрическая проницаемость оксида, $\varepsilon_{0}-$ диэлектрическая постоянная $\left(8.85 \cdot 10^{-3} \Pi \Phi / \mathrm{M \kappa M}\right), d_{o x}-$ толщина оксидного слоя, $A-$ площадь электрода. Диэлектрическая проницаемость равна 19 , что намного ниже, чем в случае безводного $h-\mathrm{La}_{2} \mathrm{O}_{3}(\varepsilon=27)$, но больше, чем $\varepsilon=4$ для $\mathrm{SiO}_{2}$.

\section{4. Заключение}

В данной работе исследованы проводящие $(\sigma-V)$ и емкостные $(C-V)$-характеристики поверхностногидрированных пленок $\mathrm{OH}-\mathrm{La}_{2} \mathrm{O}_{3}$, напыленных на кремниевые подложки с р-типом проводимости. Выявлена ОДП в структурах $\mathrm{Al} / \mathrm{OH}-\mathrm{La}_{2} \mathrm{O}_{3} / p$ - $\mathrm{Si}$, которая исчезает при хранении пленок в вакуумной камере. ОДП восстанавливается путем низковакуумного отжига при $650^{\circ} \mathrm{C}$. Наблюдается хорошая корреляция между ИК поглощением -OH-групп и электрическими характеристиками. Происхождение ОДП объясняется переносом протонов по цепочкам молекул воды, связанных водородными связями на поверхности пленки $\mathrm{OH}-\mathrm{La}_{2} \mathrm{O}_{3}$.

\section{Финансирование работы}

Работа выполнена при поддержке Российско-Армянского университета по постановлению Министерства образования и науки РФ.

\section{Конфликт интересов}

Авторы заявляют, что у них нет конфликта интересов.

\section{Список литературы}

[1] C.H. Hsu, M.T. Wang, J. Ya-Min Lee. J. Appl. Phys., 100, 074108 (2006).

[2] K. Xiong, J. Robertson. Appl. Phys. Lett., 95, 022903 (2009).

[3] Y. Wang, R. Jia, C. Li, Y. Zhang. AIP Adv., 5, 087166 (2015).

[4] M. Gutowski, J.E. Jaffe, C.-L. Liu, M. Stoker, R.I. Hegde, R.S. Rai, P.J. Tobin. Appl. Phys. Lett., 80, 1897 (2002).

[5] Liu Qi-Ya, Fang Ze-Bo, Ji Ting, Liu Shi-Yan, Tan Yong-Sheng, Chen Jia-Jun, Zhu Yan-Yan. Chin. Phys. Lett., 31, 027702 (2014).

[6] C. Yang, H. Fan, Sh. Qiu, Y. Xi, Y. Fu. Surf. Rev. Lett., 15, 271 (2008).

[7] Lin Chen, Wen Yang, Ye Li, Qing-Qing Sun, Peng Zhou, Hong-Liang Lu, Shi-Jin Ding, D. Wei Zhang. J. Vac. Sci. Technol. A, 30, 01 A148 (2012).

[8] H. Zhao, H. Tu, H.F. Wei, Y. Xiong, X. Zhang, J. Du. Phys. Status Solidi (RRL), 7, 1005 (2013).

[9] Y. Zhao, M. Toyama, K. Kita, K. Kyuno, A. Toriumi. Appl. Phys. Lett., 88, 072904 (2006).

[10] A. Igityan, N. Aghamalyan, S. Petrosyan, I. Gambaryan, G. Badalyan, R. Hovsepyan, Y. Kafadaryan. Appl. Phys. A, 123, 448 (2017).

[11] A.Š. Vuk, R. Ješe, B. Orel, G. Dražic. IJP, 7, 163 (2005).

[12] L. Esaki. Phys. Rev., 109, 603 (1958).

[13] J. Chen, M.A. Reed, A.M. Rawlett, J.M. Tour. Science, 286, 1550 (1999).

[14] N.A. Zimbovskaya, M.R. Pederson. Phys. Rev. B, 78, 153105 (2008).

[15] Q. Tang, H.K. Moon, Y. Lee, S.M. Yoon, H.J. Song, H. Lim, H.C. Choi. J. Am. Chem. Soc., 129, 11018 (2007).

[16] H.K. Lee, M.H.C. Jin. Appl. Phys. Lett., 97, 013306 (2010).

[17] D. Joung, L. Anjia, H. Matsui, S.I. Khondaker. Appl. Phys. A: Mater. Sci. Process, 112, 305 (2013).

[18] Song Hi Lee, Jayendran C. Rasaiah. J. Chem. Phys., 135, 124505 (2011).

[19] Takeo Miyake, Marco Rolandi. J. Phys.: Condens. Matter, 28, 023001 (2016).

[20] S.M. Sze. Physics of Semiconductor Devices (Wiley, N.Y., 1969).

[21] Т.В. Бланк, Ю.А. Гольдберг. ФТП, 41, 1281 (2007).

[22] M.A. Lampert. Phys. Rev., 103, 1648 (1956).

[23] Л.П. Павлов. Методы измерения параметров полупроводниковых материалов (М., Высш. шк., 1987).

Редактор Г.А. Оганесян 


\title{
Negative differential conductivity in lanthanum oxide based structures
}

\author{
A. Igityan ${ }^{1,2}$, N. Aghamalyan ${ }^{1,2}$, R. Hovsepyan ${ }^{1,2}$, \\ S. Petrosyan 1,2, G. Badalyan ${ }^{1}$, I. Gambaryan ${ }^{1}$, \\ A. Papikyan ${ }^{1}$, Y. Kafadaryan ${ }^{\mathbf{1 , 2}}$ \\ ${ }^{1}$ Institute for Physical Research \\ of National Academy of Sciences of Armenia, \\ 0203 Ashtarak, Armenia \\ ${ }^{2}$ Russian-Armenian University, \\ 0051 Yerevan, Armenia
}

Abstract Using the method of electron beam evaporation, transparent surface-hydrogenated lanthanum oxide films $\left(\mathrm{OH}-\mathrm{La}_{2} \mathrm{O}_{3}\right)$ with a thickness of 40,140 , and $545 \mathrm{~nm}$ were prepared. The electrical and optical characteristics of $\mathrm{Al} / \mathrm{OH}-\mathrm{La}_{2} \mathrm{O}_{3} / p$-Si structures were studied, where aluminum and a silicon substrate with $p$-type conductivity were used as the upper and lower electrodes, respectively. A region of negative differential conductivity (NDC) was found on the voltage dependence of conductivity at forward bias; the possible mechanism of NDC is explained by proton transfer along chains of water molecules bound by hydrogen bonds on the surface of an $\mathrm{OH}-\mathrm{La}_{2} \mathrm{O}_{3}$ film. 